\title{
On the well-posedness of the incompressible flow in porous media
}

Fuyi $\mathrm{Xu}^{\mathrm{a}, \mathrm{b}, *}$, Lishan Liu ${ }^{\mathrm{a}}$

${ }^{a}$ School of Mathematics Science, Qufu normal University, Qufu, 263516, China.

${ }^{b}$ School of Science, Shandong University of Technology, Zibo, 255049, China.

Communicated by R. Saadati

\begin{abstract}
In this paper, we are concerned with the two-dimensional (2D) incompressible flow in porous media (IPM) in the whole space. We prove the local well-posedness of the solutions for the system in Besov spaces of weak type and obtain blow-up criterion of solutions by particle trajectory method and Fourier localization method. (C)2016 All rights reserved.
\end{abstract}

Keywords: Well-posedness, blow-up criterion, particle trajectory mapping, Fourier localization method, Besov spaces of weak type.

2010 MSC: 35Q35, 76B03.

\section{Introduction and preliminaries}

In this paper, we consider the following two-dimensional (2D) incompressible flow in porous media (IPM):

$$
\begin{cases}\theta_{t}+u \cdot \nabla \theta=0, & (x, t) \in \mathbb{R}^{2} \times(0, \infty), \\ u=-k(\nabla p+g \gamma \theta), & (x, t) \in \mathbb{R}^{2} \times(0, \infty), \\ \nabla \cdot u=0, & (x, t) \in \mathbb{R}^{2} \times(0, \infty), \\ \theta(x, 0)=\theta_{0}(x), & x \in \mathbb{R}^{2},\end{cases}
$$

where $\theta$ is the liquid temperature, $k$ is the matrix position-independent medium permeabilities in different directions respectively divided by the viscosity, $g$ is the acceleration due to gravity, and the vector $\gamma=(0,1)$.

\footnotetext{
${ }^{*}$ Corresponding author

Email addresses: zbxufuyi@163.com (Fuyi Xu), mathlls@163.com (Lishan Liu)
} 
Moreover, $p$ is the liquid pressure and $u$ represents the liquid discharge by Darcy's law. For simplicity, we set $k=g=1$. It is well-known that the dynamics of a fluid through a porous medium is a complex and not thoroughly understood phenomenon [2, 9]. In real applications, one might be interested in the transport of a dissolved contaminant in porous media where the contaminant is convected with the subsurface water [10. For example, one is usually interested in the time taken by the pollutant to reach the water table below. Such flows also occur in artificial recharge wells where water and (or) chemicals from the surface are transported into aquifers. In this case, the chemicals may be the nutrients required for degradation of harmful polluting hydrocarbons resident in the aquifer after a spillage.

By rewriting Darcy's law, we obtain the expression of velocity $u$ only in terms of temperature $\theta$, see for example [8]. Due to the incompressibility, by taking the curl operator first and the $\nabla^{\perp}:=\left(-\partial_{x_{2}}, \partial_{x_{1}}\right)$ operator second on both sides of Darcy's law, we have

$$
-\Delta u=\nabla^{\perp}\left(\partial_{x_{1}} \theta\right)=\left(-\partial_{x_{1}} \partial_{x_{2}} \theta, \partial_{x_{1}}^{2} \theta\right),
$$

thus the velocity $u$ can be recovered as

$$
u(x, t)=-\frac{1}{2 \pi} \int_{\mathbb{R}^{2}} \ln |x-y| \frac{\partial \theta}{\partial y_{1}}(y, t) d y, x \in \mathbb{R}^{2} .
$$

Thus,

$$
u(x, t)=-\frac{1}{2}(0, \theta(x, t))+P V \int_{\mathbb{R}^{2}} H(x-y) \theta(y, t) d y, x \in \mathbb{R}^{2},
$$

where the kernel $H(\cdot)$ is defined by

$$
H(x)=\frac{1}{2 \pi}\left(-2 \frac{x_{1} x_{2}}{|x|^{4}}, \frac{x_{1}^{2}-x_{2}^{2}}{|x|^{4}}\right) .
$$

In general, each coefficient of $u(\cdot, t)$ (with $t$ as parameter) is only the linear combination of the singular integral of $\theta$ and $\theta$ itself. We write the general version as

$$
u(x, t)=\mathcal{C}(\theta)+\mathcal{R}(\theta) .
$$

Obvious, $\mathcal{C}$ equals a constant multiplication operator, whereas $\mathcal{R}$ means a singular integral operator.

Recently, Chae studied the local well-posedness and blow-up criterion for the incompressible Euler equations in the Triebel-Lizorkin spaces [4, 5] and Besov spaces [6] respectively. As we know, Triebel-Lizorkin spaces and Besov spaces are the unification of several classical function spaces such as Lebegue spaces $L^{p}\left(\mathbb{R}^{n}\right)$, Sobolev spaces $H_{p}^{s}\left(\mathbb{R}^{n}\right)$, Lipschitz spaces $C^{s}\left(\mathbb{R}^{n}\right)$, and so on. In [12], Takada first introduced Besov type function spaces $B_{p, r}^{s, \infty}\left(\mathbb{R}^{n}\right)$, based on the weak $L^{p}$-spaces $L^{p, \infty}\left(\mathbb{R}^{n}\right)$ instead of the standard $L^{p}$-spaces, and proved the local-in-time existence and uniqueness of solutions for the Euler equations in the function spaces. Moreover, they also showed the blow-up criterion for the solution in spaces $B_{p, r}^{s, \infty}\left(\mathbb{R}^{n}\right)$. Very recently, the IPM (1.1) was introduced and investigated by Córdoba and his group [8]. In [8], the authors obtained local existence and uniqueness by the Picard Theorem and presented different global existence criterion by the BMO norm for $\nabla \theta$, where BMO is the space of functions of bounded mean oscillation (see [11]). In [13], authors obtained the well-posedness of the IPM [1.1] in Triebel-Lizorkin spaces.

The aim of this paper is to prove the local-in-time existence and uniqueness of solutions for twodimensional IPM 1.1] in Besov spaces of weak type $B_{p, r}^{s, \infty}\left(\mathbb{R}^{2}\right)$. Moreover, we also show the blow-up criterion of solutions.

Our main results now read as follows.

\section{Theorem 1.1.}

(1) (Local-in-time existence) Let $1<p<\infty$. Assume that $s$ and $r$ satisfy $s>2 / p+1,1 \leq r \leq \infty$, or $s=2 / p+1, r=1$. Suppose that $\theta_{0} \in B_{p, r}^{s, \infty}\left(\mathbb{R}^{2}\right)$. Then there exists $T$ such that the IPM (1.1) has a unique solution $\theta \in C\left([0, T) ; B_{p, r}^{s, \infty}\left(\mathbb{R}^{2}\right)\right)$. 
(2) (Blow-up criterion) Let $1<p<\infty$.

(i) (Super-critical case) Let $s>2 / p+1,1 \leq r \leq \infty$, the local-in-time solution $\theta \in C\left([0, T) ; B_{p, r}^{s, \infty}\left(\mathbb{R}^{2}\right)\right)$ constructed in IPM (1.1) blows up at $T^{*}>T$ in $B_{p, r}^{s, \infty}\left(\mathbb{R}^{2}\right)$, namely

$$
\limsup _{t \nearrow T^{*}}\|\theta\|_{B_{p, r}^{s, \infty}\left(\mathbb{R}^{2}\right)}=+\infty, \quad T^{*}<\infty
$$

if and only if

$$
\int_{0}^{T^{*}}\|\nabla \theta\|_{\dot{B}_{\infty, \infty}^{0}} d t=+\infty
$$

(ii) (Critical case) Let $s=2 / p+1, r=1$, the local-in-time solution $\theta \in C\left([0, T) ; B_{p, 1}^{2 / p+1, \infty}\left(\mathbb{R}^{2}\right)\right)$ constructed in IPM (1.1) blows up at $T^{*}>T$ in $B_{p, 1}^{2 / p+1, \infty}\left(\mathbb{R}^{2}\right)$, namely

$$
\limsup _{t \nearrow T^{*}}\|\theta\|_{B_{p, 1}^{2 / p+1, \infty}\left(\mathbb{R}^{2}\right)}=+\infty, \quad T^{*}<\infty
$$

if and only if

$$
\int_{0}^{T^{*}}\|\nabla \theta\|_{\dot{B}_{\infty, 1}^{0}} d t=+\infty
$$

Remark 1.2. By using the continuous embedding relations, $L^{\infty} \hookrightarrow \mathrm{BMO} \hookrightarrow \dot{B}_{\infty, \infty}^{0}$ and $B_{p, r}^{s}\left(\mathbb{R}^{2}\right) \hookrightarrow$ $B_{p, r}^{s, \infty}\left(\mathbb{R}^{2}\right)$, the results from Theorem 1.1 (2) improve the existing blow-up criterion in [8].

Remark 1.3. By the same method, we can get the local well-posedness and the blow-up criterion of the smooth solutions for inviscid two-dimensional (2D) quasi-geostrophic equations.

\section{Preliminaries and lemmas}

In this section, we give definitions and some properties of the weak space in the same way as the usual Besov space is treated.

Firstly, let us recall the weak $L^{p}$-space $L^{p, \infty}\left(\mathbb{R}^{n}\right)$ and the Littlewood- Paley operators $\Delta_{(0)},\left\{\dot{\Delta}_{j}\right\}_{j \in \mathbb{Z}}$. For a measurable function $f$ on $\mathbb{R}^{n}$, the average function $f^{* *}(t)$ is defined by

$$
f^{* *}(t)=\frac{1}{t} \int_{0}^{t} f^{*}(s) d s
$$

for $t>0$, where $f^{*}$ denotes the usual non-increasing rearrangement of $f$.

Definition 2.1. For $1 \leq p \leq \infty$, the weak $L^{p}$-space $L^{p, \infty}\left(\mathbb{R}^{n}\right)$ is defined as the set of the measurable functions $f$ on $\mathbb{R}^{n}$ such that

$$
\|f\|_{L^{p, \infty}}=\sup _{t>0}\left(t^{\frac{1}{p}} f^{* *}(t)\right)<\infty .
$$

Let $\chi(t)$ be a smooth function on $[0, \infty)$ such that $0 \leq \chi(t) \leq 1, \chi(t) \equiv 1$ for $0 \leq t \leq 3 / 2$ and $\operatorname{supp} \chi \subset[0,5 / 3)$, and we set $\mathcal{F}\left[\varphi_{j}\right](\xi)=\chi\left(2^{-j}|\xi|\right)-\chi\left(2^{1-j}|\xi|\right)$ and $\mathcal{F}\left[\varphi_{0}\right](\xi)=\chi(|\xi|)$ for $\xi \in \mathbb{R}^{n}$, where $\mathcal{F} f=\widehat{f}$ denotes the Fourier transform of $f$ on $\mathbb{R}^{n}$. Let $\mathcal{S}\left(\mathbb{R}^{n}\right)$ be the Schwartz class of rapidly decreasing function. By given $f \in \mathcal{S}^{\prime}\left(\mathbb{R}^{n}\right)$, we denote $\Delta_{(0)} f=\varphi_{0} * f$, and $\dot{\Delta}_{j} f=\varphi_{j} * f$. Then, we introduce the inhomogeneous weak $B_{p, r}^{s, \infty}\left(\mathbb{R}^{n}\right)$ and the homogeneous weak space $\dot{B}_{p, r}^{s, \infty}\left(\mathbb{R}^{n}\right)$ by the following definition.

Definition 2.2. For $1<p<\infty, 1 \leq r \leq \infty$ and $s \in \mathbb{R}$, the inhomogeneous Besov space of weak type $B_{p, r}^{s, \infty}\left(\mathbb{R}^{n}\right)$ is defined by

where

$$
B_{p, r}^{s, \infty}=\left\{f \in \mathcal{S}^{\prime}\left(\mathbb{R}^{n}\right):\|f\|_{B_{p, r}^{s, \infty}}<+\infty\right\}
$$

$$
\|f\|_{B_{p, r}^{s, \infty}}=\left\|\Delta_{(0)} f\right\|_{L^{p, \infty}}+\left\{\begin{array}{l}
\left(\sum_{j=1}^{\infty} 2^{j s r}\left\|\Delta_{j} f\right\|_{L^{p, \infty}}^{r}\right)^{\frac{1}{r}}, \quad 1 \leq r<\infty, \\
\sup _{j \in \mathbb{Z}} 2^{j s}\left\|\Delta_{j} f\right\|_{L^{p, \infty}}, \quad r=\infty .
\end{array}\right.
$$


Definition 2.3. For $1<p<\infty, 1 \leq r \leq \infty$ and $s \in \mathbb{R}$, the homogeneous Besov space of weak type $\dot{B}_{p, r}^{s, \infty}\left(\mathbb{R}^{n}\right)$ is defined by

$$
\dot{B}_{p, r}^{s, \infty}=\left\{\mathbb{Z}^{\prime}\left(\mathbb{R}^{n}\right):\|f\|_{\dot{B}_{p, r}^{s, \infty}}<+\infty\right\},
$$

where

$$
\|f\|_{\dot{B}_{p, r}^{s, \infty}}=\left\{\begin{array}{l}
\left(\sum_{j \in \mathbb{Z}} 2^{j s r}\left\|\Delta_{j} f\right\|_{L^{p, \infty}}^{r}\right)^{\frac{1}{r}}, \quad 1 \leq r<\infty, \\
\sup _{j \in \mathbb{Z}} 2^{j s}\left\|\Delta_{j} f\right\|_{L^{p, \infty}}, \quad r=\infty,
\end{array}\right.
$$

where the space $\mathbb{Z}^{\prime}\left(\mathbb{R}^{n}\right)$ by the dual space of $\mathbb{Z}\left(\mathbb{R}^{n}\right)=\left\{f \in \mathcal{S}\left(\mathbb{R}^{n}\right): D^{\alpha} \hat{f}(0)=0\right.$, where $\alpha$ is multi-index $\}$.

It is easy to see that both $B_{p, r}^{s, \infty}\left(\mathbb{R}^{n}\right)$ and $\dot{B}_{p, r}^{s, \infty}\left(\mathbb{R}^{n}\right)$ are Banach spaces. Recall that the standard Besov space $B_{p, r}^{s}\left(\mathbb{R}^{n}\right)$ and the homogeneous Besov space $\dot{B}_{p, r}^{s}\left(\mathbb{R}^{n}\right)$ are defined in the same way as $B_{p, r}^{s, \infty}\left(\mathbb{R}^{n}\right)$ and $\dot{B}_{p, r}^{s, \infty}\left(\mathbb{R}^{n}\right)$ respectively, based on the weak $L^{p}$-spaces $L^{p, \infty}\left(\mathbb{R}^{n}\right)$ instead of the standard $L^{p}$-spaces.

Next, we show some properties of weak space.

Lemma 2.4 ([7]). (Bernstein's inequalities) Let $k \in \mathbb{N}$ and assume $f \in L^{p}\left(\mathbb{R}^{n}\right), 1 \leq p \leq \infty$, and

$$
\operatorname{supp} \widehat{f} \subset\left\{\xi \in \mathbb{R}^{n}: 2^{j-1} \leq|\xi| \leq 2^{j+1}\right\} .
$$

Then there exist constants $C_{k}, C_{k}^{\prime}$ independent of $f, j$ such that the following inequalities hold

$$
C_{k}^{\prime} 2^{j k}\|f\|_{L^{p}} \leq \sup _{|\alpha|=k}\left\|\partial^{\alpha} f\right\|_{L^{p}} \leq C_{k} 2^{j k}\|f\|_{L^{p}} .
$$

Similarly, we have the following Bernstein type Lemma also for $L^{p, \infty}\left(\mathbb{R}^{n}\right)$.

Lemma 2.5 ([7]). Let $k \in \mathbb{N}$ and assume $f \in L^{p, \infty}\left(\mathbb{R}^{n}\right), 1<p<\infty$, and

$$
\operatorname{supp} \widehat{f} \subset\left\{\xi \in \mathbb{R}^{n}: 2^{j-1} \leq|\xi| \leq 2^{j+1}\right\} .
$$

Then there exist constants $C_{p, k}, C_{p, k}^{\prime}$ such that the following inequalities hold

$$
C_{p, k}^{\prime} 2^{j k}\|f\|_{L^{p, \infty}} \leq \sup _{|\alpha|=k}\left\|\partial^{\alpha} f\right\|_{L^{p, \infty}} \leq C_{p, k} 2^{j k}\|f\|_{L^{p, \infty}} .
$$

As an immediate consequence of Lemma 2.5, we also have the following results.

Lemma 2.6. Let $k \in \mathbb{N}$. Assume $s \in \mathbb{R}, 1<p<\infty, 1 \leq r \leq \infty$, the following inequalities hold

$$
C_{k}^{\prime}\|f\|_{\dot{B}_{p, r}^{s+k, \infty}} \leq \sum_{i=1}^{n}\left\|\partial_{i}^{k} f\right\|_{\dot{B}_{p, r}^{s, \infty}} \leq C_{k}\|f\|_{\dot{B}_{p, r}^{s+k, \infty}} .
$$

We next investigate the relations between the homogeneous and the inhomogeneous spaces.

Lemma 2.7. Assume $s>0,1<p<\infty, 1 \leq r \leq \infty$, then the following relations hold

$$
B_{p, r}^{s, \infty}\left(\mathbb{R}^{n}\right)=L^{p, \infty}\left(\mathbb{R}^{n}\right) \cap \dot{B}_{p, r}^{s, \infty}\left(\mathbb{R}^{n}\right), \quad\|f\|_{B_{p, r}^{s, \infty}} \sim\|f\|_{L^{p, \infty}}+\|f\|_{\dot{B}_{p, r}^{s, \infty}}
$$

Since the proof of Lemma 2.7 is standard, we may omit it. For the details, see Bergh and Löfstrom [3].

Lemma 2.8 ([12]). For $j \in \mathbb{Z}, 1<p<\infty$, we have

$$
\left\|\dot{\Delta}_{j} f\right\|_{L^{\infty}} \leq C 2^{j n / p}\left\|\dot{\Delta}_{j} f\right\|_{L^{p, \infty}}, \quad\left\|\Delta_{(0)} f\right\|_{L^{\infty}} \leq C\left\|\Delta_{(0)} f\right\|_{L^{p, \infty}} .
$$


Lemma 2.9 ([12]). Assume $s \in \mathbb{R}, 1<p<\infty, 1 \leq r \leq \infty$, the following relations hold

$$
\dot{B}_{p, r}^{s, \infty}\left(\mathbb{R}^{n}\right) \hookrightarrow \dot{B}_{\infty, r}^{s-n / p}\left(\mathbb{R}^{n}\right), \quad B_{p, r}^{s, \infty}\left(\mathbb{R}^{n}\right) \hookrightarrow B_{\infty, r}^{s-n / p}\left(\mathbb{R}^{n}\right) .
$$

Furthermore, assume $s, r$ satisfy $s>n / p, 1 \leq r \leq \infty$ or $s=n / p, r=1$, we have

$$
B_{p, r}^{s, \infty}\left(\mathbb{R}^{n}\right) \hookrightarrow L^{\infty}\left(\mathbb{R}^{n}\right) .
$$

Finally, for the proof of local existence and blow-up criterion of the smooth solutions, we need to show the following estimates.

Proposition $2.10([12])$. Let $s>0,(p, r) \in(1, \infty) \times(1, \infty]$, or $p=r=\infty$, then there exists a constant $C$ such that

$$
\begin{aligned}
& \|f g\|_{\dot{B}_{p, r}^{s, \infty}} \leq C\left(\|f\|_{L^{\infty}}\|g\|_{\dot{B}_{p, r}^{s, \infty}}+\|g\|_{L^{\infty}}\|f\|_{\dot{B}_{p, r}^{s, \infty}}\right), \\
& \|f g\|_{B_{p, r}^{s, \infty}} \leq C\left(\|f\|_{L^{\infty}}\|g\|_{B_{p, r}^{s, \infty}}+\|g\|_{L^{\infty}}\|f\|_{B_{p, r}^{s, \infty}}\right) .
\end{aligned}
$$

Proposition 2.11. For $1<p<\infty, s>\frac{n}{p}, 1 \leq r \leq \infty$, there is a constant $C=C(p, s, r, n)$ such that

$$
\|f\|_{L^{\infty}} \leq C\left\{1+\|f\|_{\dot{B}_{\infty, \infty}^{0}}\left(\log ^{+}\|f\|_{B_{p, r}^{s, \infty}}+1\right)\right\}
$$

holds for all $f \in B_{p, r}^{s, \infty}\left(\mathbb{R}^{n}\right)$.

The proof is similar to the inequality of the Beale-Kato-Majda type in [1], here we omit it.

Proposition $2.12([12])$. Let $(p, r) \in(1, \infty) \times[1, \infty], s>0$, there exists a constant $C=C(p, s, r, n)$ such that

$$
\begin{gathered}
\left.\left(\sum_{j \in \mathbb{Z}} 2^{s j r} \|\left[v, \Delta_{j}\right] \cdot \nabla \theta\right) \|_{L^{p, \infty}}^{r}\right)^{\frac{1}{r}} \leq C\left(\|\nabla v\|_{\infty}\|\theta\|_{\dot{B}_{p, r}^{s, \infty}}+\|\nabla \theta\|_{\infty}\|v\|_{\dot{B}_{p, r}^{s, \infty}}\right), \\
\left.\sup _{j \in \mathbb{Z}} 2^{s j} \|\left[v, \Delta_{j}\right] \cdot \nabla \theta\right) \|_{L^{p, \infty}} \leq C\left(\|\nabla v\|_{\infty}\|\theta\|_{\dot{B}_{p, r}^{s, \infty}}+\|\nabla \theta\|_{\infty}\|v\|_{\dot{B}_{p, r}^{s, \infty}}\right) .
\end{gathered}
$$

Proposition $2.13([12])$. Let $(p, r) \in(1, \infty) \times[1, \infty], s>0$, there is a constant $C=C(p, s, r, n)$ such that

$$
\begin{gathered}
\left.\left(\sum_{j \in \mathbb{Z}} 2^{s j r} \|\left[v, \Delta_{j}\right] \cdot \nabla \theta\right) \|_{L^{p, \infty}}^{r}\right)^{\frac{1}{r}} \leq C\left(\|\nabla v\|_{\infty}\|\theta\|_{\dot{B}_{p, r}^{s, \infty}}+\|\theta\|_{\infty}\|v\|_{\dot{B}_{p, r}^{s+1, \infty}}\right), \\
\left.\sup _{j \in \mathbb{Z}} 2^{s j} \|\left[v, \Delta_{j}\right] \cdot \nabla \theta\right) \|_{L^{p, \infty}} \leq C\left(\|\nabla v\|_{\infty}\|\theta\|_{\dot{B}_{p, r}^{s, \infty}}+\|\theta\|_{\infty}\|v\|_{\dot{B}_{p, r}^{s+1, \infty}}\right) .
\end{gathered}
$$

\section{The proof of main results}

In this section, we divide the proof of Theorem 1.1 into five steps as follows.

Proof of Theorem 1.1.

Step 1: A priori estimate.

By taking the operation $\Delta_{j}$ on both sides of (1.1), we get

$$
\partial_{t} \Delta_{j} \theta+u \cdot \nabla \Delta_{j} \theta=\left[u, \Delta_{j}\right] \cdot \nabla \theta,
$$

where we denote the commutator $\left[u, \Delta_{j}\right] \cdot \nabla \theta=u \cdot \nabla \Delta_{j} \theta-\Delta_{j}(u \cdot \nabla \theta)$. Let $X(\alpha, t)$ be the particle trajectory mapping defined by the solutions of the following ordinary differential equations

$$
\left\{\begin{array}{l}
\frac{d}{d t} X(\alpha, t)=u(X(\alpha, t), t), \\
X(\alpha, 0)=\alpha .
\end{array}\right.
$$


We note that $X(\alpha, t)$ is a volume-preserving diffeomorphism due to $\operatorname{div} u=0$. Then by (3.1), we have

$$
\frac{d}{d t} \Delta_{j} \theta(X(\alpha, t), t)=\left[u, \Delta_{j}\right] \cdot \nabla \theta(X(\alpha, t), t) .
$$

Integrating from 0 to $t$ yields

$$
\Delta_{j} \theta(X(\alpha, t), t)=\Delta_{j} \theta_{0}(\alpha)+\int_{0}^{t}\left[u, \Delta_{j}\right] \cdot \nabla \theta(X(\alpha, \tau), \tau) d \tau .
$$

Since the map $\alpha \rightarrow X(\alpha, t)$ is volume-preserving for all $t$, by taking the $L^{p, \infty}$-norm of both sides of $(3.3)$, we have

$$
\left\|\Delta_{j} \theta\right\|_{L^{p, \infty}} \leq C_{p}\left\|\Delta_{j} \theta_{0}\right\|_{L^{p, \infty}}+C_{p} \int_{0}^{t}\left\|\left[u, \Delta_{j}\right] \cdot \nabla \theta\right\|_{L^{p, \infty}} d \tau .
$$

By multiplying both sides of (3.4) by $2^{j s}$ and then taking the $l^{r}$-norm, we obtain from Minkowski's inequality

$$
\|\theta\|_{\dot{B}_{p, r}^{s, \infty}} \leq C_{p}\left\|\theta_{0}\right\|_{\dot{B}_{p, r}^{s, \infty}}+\left\{\begin{array}{l}
C_{p} \int_{0}^{t}\left\|\left[u, \Delta_{j}\right] \cdot \nabla \theta\right\|_{\dot{B}_{p, r}^{s, \infty}} d \tau, \quad 1 \leq r<\infty, \\
C_{p} \int_{0}^{t} \sup _{j \in \mathbb{Z}} 2^{s j}\left\|\left[u, \Delta_{j}\right] \cdot \nabla \theta\right\|_{L^{p, \infty}} d \tau, \quad r=\infty .
\end{array}\right.
$$

Then, for $1 \leq r<\infty$, it follows from Proposition 2.12 that

$$
\begin{aligned}
\left\|\left[u, \Delta_{j}\right] \cdot \nabla \theta\right\|_{\dot{B}_{p, r}^{s, \infty}} & \leq C\left(\|\nabla u\|_{\infty}\|\theta\|_{\dot{B}_{p, r}^{s, \infty}}+\|\nabla \theta\|_{\infty}\|u\|_{\dot{B}_{p, r}^{s, \infty}}\right) \\
& \leq C\left(\|\nabla u\|_{\infty}+\|\nabla \theta\|_{\infty}\right)\|\theta\|_{\dot{B}_{p, r}^{s, \infty}},
\end{aligned}
$$

and for $r=\infty$,

$$
\begin{aligned}
\sup _{j \in \mathbb{Z}} 2^{s j}\left\|\left[u, \Delta_{j}\right] \cdot \nabla \theta\right\|_{L^{p, \infty}} & \leq C\left(\|\nabla u\|_{\infty}\|\theta\|_{\dot{B}_{p, r}^{s, \infty}}+\|\nabla \theta\|_{\infty}\|u\|_{\dot{B}_{p, r}^{s, \infty}}\right) \\
& \leq C\left(\|\nabla u\|_{\infty}+\|\nabla \theta\|_{\infty}\right)\|\theta\|_{\dot{B}_{p, r}^{s, \infty}}
\end{aligned}
$$

where we used the facts that $u(x, t)=\mathcal{C}(\theta)+\mathcal{R}(\theta)$ and that singular integral operator is bounded on $\dot{B}_{p, r}^{s, \infty}$. Thus, we have

$$
\|\theta\|_{\dot{B}_{p, r}^{s, \infty}} \leq C_{p}\left\|\theta_{0}\right\|_{\dot{B}_{p, r}^{s, \infty}}+C_{p} \int_{0}^{t}\left(\|\nabla u\|_{\infty}+\|\nabla \theta\|_{\infty}\right)\|\theta\|_{\dot{B}_{p, r}^{s, \infty}} d \tau .
$$

This together with Gronwall's inequality gives a priori estimate in the homogeneous $\dot{B}_{p, r}^{s, \infty}$

$$
\|\theta\|_{\dot{B}_{p, r}^{s, \infty}} \leq C\left\|\theta_{0}\right\|_{\dot{B}_{p, r}^{s, \infty}} \exp \left(C \int_{0}^{t}\left(\|\nabla u\|_{\infty}+\|\nabla \theta\|_{\infty}\right) d \tau\right) .
$$

Moreover, to derive the estimate in the inhomogeneous space $B_{p, r}^{s, \infty}$, we need to handle $\|\theta\|_{L^{p, \infty}}$. We obtain from 1.1 and 3.2

$$
|\theta(X(\alpha, t), t)| \leq|\theta(\alpha, 0)|,
$$

which yields with the aid of the fact that $\operatorname{det} \nabla_{\alpha} X(\alpha, t)=1$ that

$$
\|\theta\|_{L^{p, \infty}} \leq C_{p}\left\|\theta_{0}\right\|_{L^{p, \infty}} .
$$

By adding (3.5) to (3.6), we obtain the inhomogeneous space estimate

$$
\|\theta\|_{B_{p, r}^{s, \infty}} \leq C_{p}\left\|\theta_{0}\right\|_{B_{p, r}^{s, \infty}}+C_{p} \int_{0}^{t}\left(\|\nabla u\|_{\infty}+\|\nabla \theta\|_{\infty}\right)\|\theta\|_{B_{p, r}^{s, \infty}} d \tau .
$$

By Gronwall's inequality, we obtain a priori estimate in the inhomogeneous spaces

$$
\|\theta\|_{B_{p, r}^{s, \infty}} \leq C\left\|\theta_{0}\right\|_{B_{p, r}^{s, \infty}} \exp \left(C \int_{0}^{t}\left(\|\nabla u\|_{\infty}+\|\nabla \theta\|_{\infty}\right) d \tau\right) .
$$


Step 2: Approximate solutions and uniform estimates.

To obtain the approximate solutions, we first set $\left(\theta^{0}, u^{0}\right)=(0,0)$ and then define $\left\{u^{m}, \theta^{m}\right\}_{m \in \mathbb{N}}$ as the solutions of the following linear equations:

$$
\begin{cases}\theta_{t}^{m+1}+u^{m} \cdot \nabla \theta^{m+1}=0, & (x, t) \in \mathbb{R}^{2} \times(0, \infty), \\ u^{m}=\mathcal{C}\left(\theta^{m}\right)+\mathcal{S}\left(\theta^{m}\right), & (x, t) \in \mathbb{R}^{2} \times(0, \infty), \\ \nabla \cdot u^{m}=0, & (x, t) \in \mathbb{R}^{2} \times(0, \infty), \\ \theta^{m+1}(x, 0)=P_{m+3} \theta_{0}(x), & x \in \mathbb{R}^{2}\end{cases}
$$

Similar to Step 1, let $X^{m}(\alpha, t)$ be the particle trajectory mapping defined by the solutions of the following ordinary differential equations:

$$
\left\{\begin{array}{l}
\frac{d}{d t} X^{m}(\alpha, t)=u^{m}\left(X^{m}(\alpha, t), t\right), \\
X^{m}(\alpha, 0)=\alpha,
\end{array}\right.
$$

for each $n \in \mathbb{N}$. Then, by following the same procedure of estimate leading to (3.7), we obtain

$$
\begin{aligned}
\left\|\theta^{m+1}(t)\right\|_{B_{p, r}^{s, \infty} \leq} & C\left\|\theta_{0}\right\|_{B_{p, r}^{s, \infty}} \\
& +\int_{0}^{t}\left(\left\|\nabla u^{m}(\tau)\right\|_{\infty}\left\|\theta^{m+1}(\tau)\right\|_{\dot{B}_{p, r}^{s, \infty}}+\left\|\nabla \theta^{m+1}(\tau)\right\|_{\infty}\left\|u^{m}(\tau)\right\|_{\dot{B}_{p, r}^{s, \infty}}\right) d \tau \\
\leq & C\left\|\theta_{0}\right\|_{B_{p, r}^{s, \infty}}+\int_{0}^{t}\left\|\theta^{m+1}(\tau)\right\|_{B_{p, r}^{s, \infty}}\left\|\theta^{m}(\tau)\right\|_{B_{p, r}^{s, \infty}} d \tau,
\end{aligned}
$$

where we have used $B_{p, r}^{s-1, \infty}\left(\mathbb{R}^{2}\right) \hookrightarrow L^{\infty}\left(\mathbb{R}^{2}\right)$ for $s>2 / p+1,1<p<\infty, 1 \leq r \leq \infty$ or $s=2 / p+1,1<$ $p<\infty, r=1$ and the boundedness of singular integral operator on $B_{p, r}^{s, \infty}\left(\mathbb{R}^{2}\right)$. It follows from Gronwall's inequality that

$$
\left\|\theta^{m+1}(t)\right\|_{B_{p, r}^{s, \infty}} \leq C\left\|\theta_{0}\right\|_{B_{p, r}^{s, \infty}} \exp \left(C \int_{0}^{t}\left\|\theta^{m}(\tau)\right\|_{B_{p, r}^{s, \infty}} d \tau\right),
$$

with some constant $\mathrm{C}$ independent of $m$. Therefore, for the initial data $\theta_{0} \in B_{p, r}^{s, \infty}\left(\mathbb{R}^{2}\right)$, if we choose $C_{1}>0$ such that $\left\|\theta_{0}\right\|_{B_{p, r}^{s, \infty}} \leq \frac{C_{1}}{2 C}$, we obtain from the standard induction argument that

$$
\left\|\theta^{m}\right\|_{L^{\infty}\left(0, T ; B_{p, r}^{s, \infty}\right)} \leq C_{1}
$$

for all $m \geq 0$, where $T$ can be chosen as

$$
\exp \left(C C_{1} T\right) \leq 2
$$

Step 3: Convergence.

Next we prove the convergence. Set

$$
\delta \theta^{m+1}=\theta^{m+1}-\theta^{m}, \quad \delta u^{m+1}=u^{m+1}-u^{m} .
$$

Then, it follows that $\delta \theta^{m+1}$ satisfies the following equations:

$$
\left\{\begin{array}{lc}
\partial_{t} \delta \theta^{m+1}+u^{m} \cdot \nabla \delta \theta^{m+1}=-\delta u^{m} \cdot \nabla \theta^{m}, & (x, t) \in \mathbb{R}^{2} \times(0, \infty), \\
u^{m}=\mathcal{C}\left(\theta^{m}\right)+\mathcal{S}\left(\theta^{m}\right), & (x, t) \in \mathbb{R}^{2} \times(0, \infty), \\
\nabla \cdot \delta u^{m+1}=\nabla \cdot u^{m}=0, & (x, t) \in \mathbb{R}^{2} \times(0, \infty), \\
\delta \theta^{m+1}(x, 0)=\Delta_{m+1} \theta_{0}(x), & x \in \mathbb{R}^{2} .
\end{array}\right.
$$

By applying the operation $\Delta_{j}$ on both sides of 3.12 , we get

$$
\partial_{t} \Delta_{j} \delta \theta^{m+1}+u^{m} \cdot \nabla \Delta_{j} \delta \theta^{m+1}=\left[u^{m}, \Delta_{j}\right] \cdot \nabla \delta \theta^{m+1}-\Delta_{j}\left(\delta u^{m} \cdot \nabla \theta^{m}\right) .
$$


Similar to (3.4), we have by $(3.9)$ and $(3.13)$ that

$$
\begin{aligned}
\left\|\Delta_{j} \delta \theta^{m+1}(t)\right\|_{L^{p, \infty}} \leq & C_{p}\left\|\Delta_{j} \delta \theta^{m+1}(0)\right\|_{L^{p, \infty}} \\
& +C_{p} \int_{0}^{t}\left\|\left[u^{m}, \Delta_{j}\right] \cdot \nabla \delta \theta^{m+1}(\tau)\right\|_{L^{p, \infty}} d \tau \\
& +C_{p} \int_{0}^{t}\left\|\Delta_{j}\left(\delta u^{m} \cdot \nabla \theta^{m}\right)(\tau)\right\|_{L^{p, \infty}} d \tau .
\end{aligned}
$$

By multiplying both sides of $(3.14)$ by $2^{j(s-1)}$ and then taking the $l^{r}$-norm, we obtain from Minkowski's inequality

$$
\begin{aligned}
\left\|\delta \theta^{m+1}(t)\right\|_{\dot{B}_{p, r}^{s-1, \infty}} \leq & C_{p}\left\|\delta \theta^{m+1}(0)\right\|_{\dot{B}_{p, r}^{s-1, \infty}} \\
& +\left\{\begin{array}{c}
C_{p} \int_{0}^{t}\left\|\left[u^{m}, \Delta_{j}\right] \cdot \nabla \delta \theta^{m+1}(\tau)\right\|_{\dot{B}_{p, r}^{s-1, \infty}} d \tau, \\
1 \leq r<\infty \\
C_{p} \int_{0}^{t} \sup _{j \in \mathbb{Z}} 2^{(s-1) j}\left\|\left[u^{m}, \Delta_{j}\right] \cdot \nabla \delta \theta^{m+1}(\tau)\right\|_{L^{p, \infty}} d \tau, \\
r=\infty \\
+C_{p} \int_{0}^{t}\left\|\delta u^{m} \cdot \nabla \theta^{m}(\tau)\right\|_{\dot{B}_{p, r}^{s-1, \infty}} d \tau \\
\triangleq I_{1}+I_{2}+I_{3} .
\end{array}\right.
\end{aligned}
$$

We first consider $I_{1}$. By Lemma 2.6, we have

$$
I_{1} \leq C 2^{-m-1}\left\|\Delta_{m+1} \theta_{0}\right\|_{\dot{B}_{p, r}^{s, \infty}} \leq C 2^{-m-1}\left\|\theta_{0}\right\|_{\dot{B}_{p, r}^{s, \infty}} \leq C 2^{-m-1}\left\|\theta_{0}\right\|_{B_{p, r}^{s, \infty}} .
$$

For the estimate of $I_{2}$, by Proposition 2.12 we have for $1 \leq r<\infty$,

$$
\begin{aligned}
\left(\sum_{j \in \mathbb{Z}} 2^{(s-1) j r}\left\|\left[u^{m}, \Delta_{j}\right] \cdot \nabla \delta \theta^{m+1}\right\|_{L^{p, \infty}}^{r}\right)^{\frac{1}{r}} & \leq C\left(\left\|\nabla u^{m}\right\|_{\infty}\left\|\delta \theta^{m+1}\right\|_{\dot{B}_{p, r}^{s-1, \infty}}+\left\|\delta \theta^{m+1}\right\|_{\infty}\left\|u^{m}\right\|_{\dot{B}_{p, r}^{s, \infty}}\right) \\
& \leq C\left\|\theta^{m}\right\|_{B_{p, r}^{s, \infty}}\left\|\delta \theta^{m+1}\right\|_{B_{p, r}^{s-1, \infty}},
\end{aligned}
$$

and for $r=\infty$,

$$
\begin{aligned}
\sup _{j \in \mathbb{Z}} 2^{(s-1) j}\left\|\left[u^{m}, \Delta_{j}\right] \cdot \nabla \delta \theta^{m+1}\right\|_{L^{p, \infty}} & \leq C\left(\left\|\nabla u^{m}\right\|_{\infty}\left\|\delta \theta^{m+1}\right\|_{\dot{B}_{p, r}^{s-1, \infty}}+\left\|\delta \theta^{m+1}\right\|_{\infty}\left\|u^{m}\right\|_{\dot{B}_{p, r}^{s, \infty}}\right) \\
& \leq C\left\|\theta^{m}\right\|_{B_{p, r}^{s, \infty}}\left\|\delta \theta^{m+1}\right\|_{B_{p, r}^{s-\infty}},
\end{aligned}
$$

which yields

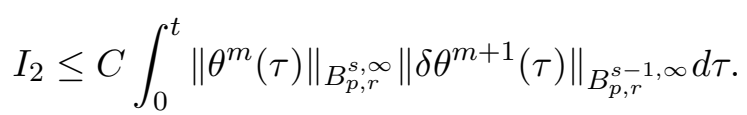

For the estimate of $I_{3}$, by 2.1 , we have

$$
\begin{aligned}
I_{3} & \leq C \int_{0}^{t}\left(\left\|\delta u^{m}(\tau)\right\|_{L^{\infty}}\left\|\nabla \theta^{m}(\tau)\right\|_{\dot{B}_{p, r}^{s-1, \infty}}+\left\|\nabla \theta^{m}(\tau)\right\|_{L^{\infty}}\left\|\delta u^{m}(\tau)\right\|_{\dot{B}_{p, r}^{s-1, \infty}}\right) d \tau \\
& \leq C \int_{0}^{t}\left\|\theta^{m}(\tau)\right\|_{B_{p, r}^{s, \infty}}\left\|\delta \theta^{m}(\tau)\right\|_{B_{p, r}^{s-1, \infty}} d \tau .
\end{aligned}
$$

By substituting (3.16), 3.17) and (3.18) into (3.15), we obtain

$$
\begin{aligned}
\left\|\delta \theta^{m+1}(t)\right\|_{\dot{B}_{p, r}^{s-1, \infty}} \leq & C 2^{-m}\left\|\theta_{0}\right\|_{B_{p, r}^{s, \infty}}+C \int_{0}^{t}\left\|\theta^{m}(\tau)\right\|_{B_{p, r}^{s, \infty}}\left\|\delta \theta^{m+1}(\tau)\right\|_{B_{p, r}^{s-1, \infty}} d \tau \\
& +C \int_{0}^{t}\left\|\theta^{m}(\tau)\right\|_{B_{p, r}^{s, \infty}}\left\|\delta \theta^{m}(\tau)\right\|_{B_{p, r}^{s-1, \infty}} d \tau .
\end{aligned}
$$


By recalling that $X^{m}(\alpha, t)$ is a solution of the ordinary differential $(3.9)$, we get

$$
\frac{d}{d t} \delta \theta^{m+1}\left(X^{m}(\alpha, t), t\right)=-\delta u^{m} \cdot \nabla \theta^{m}\left(X^{m}(\alpha, t), t\right) .
$$

By integrating the above equality from 0 to $t$ yields

$$
\delta \theta^{m+1}\left(X^{m}(\alpha, t), t\right)=\Delta_{m+1} \theta_{0}(\alpha)-\int_{0}^{t} \delta u^{m} \cdot \nabla \theta^{m}\left(X^{m}(\alpha, \tau), \tau\right) d \tau .
$$

By taking the $L^{p, \infty}$-norm on the both sides and using Minkowski's inequality, we can deduce that

$$
\left\|\delta \theta^{m+1}\right\|_{L^{p, \infty}} \leq\left\|\triangle_{m} \theta_{0}\right\|_{L^{p, \infty}}+\int_{0}^{t}\left\|\delta u^{m} \cdot \nabla \theta^{m}(\tau)\right\|_{L^{p, \infty}} d \tau .
$$

By Lemma 2.5, we have

$$
\begin{gathered}
\left\|\Delta_{m+1} \theta_{0}\right\|_{L^{p, \infty}} \leq C 2^{-m}\left\|\nabla \theta_{0}\right\|_{L^{p, \infty}} \leq C 2^{-m}\left\|\theta_{0}\right\|_{B_{p, r}^{s, \infty}}, \\
\left\|\delta u^{m} \cdot \nabla \theta^{m}\right\|_{L^{p, \infty}} \leq\left\|\delta u^{m}\right\|_{L^{p, \infty}}\left\|\nabla \theta^{m}\right\|_{L^{\infty}} \leq\left\|\delta \theta^{m}\right\|_{B_{p, r}^{s-1, \infty}}\left\|\theta^{m}\right\|_{B_{p, r}^{s, \infty}} .
\end{gathered}
$$

By substituting (3.21) and (3.22) into 3.20), we get

$$
\left\|\delta \theta^{m+1}\right\|_{L^{p, \infty}} \leq C 2^{-m-1}\left\|\theta_{0}\right\|_{B_{p, r}^{s, \infty}}+C \int_{0}^{t}\left\|\delta \theta^{m}(\tau)\right\|_{B_{p, r}^{s-1, \infty}}\left\|\theta^{m}(\tau)\right\|_{B_{p, r}^{s, \infty}} d \tau
$$

By adding (3.19) to (3.23), we derive the following estimate in the inhomogeneous space

$$
\begin{aligned}
\left\|\delta \theta^{m+1}(t)\right\|_{B_{p, r}^{s-1, \infty}} \leq & C 2^{-m}\left\|\theta_{0}\right\|_{B_{p, r}^{s, \infty}}+C \int_{0}^{T}\left\|\theta^{m}(\tau)\right\|_{B_{p, r}^{s, \infty}}\left\|\delta \theta^{m+1}(\tau)\right\|_{B_{p, r}^{s-\infty}} d \tau \\
& +C \int_{0}^{T}\left\|\theta^{m}(\tau)\right\|_{B_{p, r}^{s, \infty}}\left\|\delta \theta^{m}(\tau)\right\|_{B_{p, r}^{s-1, \infty}} d \tau
\end{aligned}
$$

for all $t \in[0, T]$, where $T$ is the same as in (3.11). Hence by (3.10) and (3.24), we have

$$
\begin{aligned}
\left\|\delta \theta^{m+1}\right\|_{L^{\infty}\left(0, T ; B_{p, r}^{s-1, \infty}\right)} \leq & C_{1} C 2^{-m-1}+C_{1} C T\left\|\delta \theta^{m+1}\right\|_{L^{\infty}\left(0, T ; B_{p, r}^{s-1, \infty}\right)} \\
& +C_{1} C T\left\|\delta \theta^{m}\right\|_{L^{\infty}\left(0, T ; B_{p, r}^{s-1, \infty}\right)} .
\end{aligned}
$$

So, if we choose $T_{1} \leq T$ sufficiently small so that $C_{1} C T_{1} \leq \frac{1}{4}$, then it follows from 3.25 that

$$
\begin{aligned}
\left\|\delta \theta^{m+1}\right\|_{L^{\infty}\left(0, T_{1} ; B_{p, r}^{s-1, \infty}\right)} \leq & C_{1} C 2^{-m-1}+\frac{1}{2}\left\|\delta \theta^{m+1}\right\|_{L^{\infty}\left(0, T_{1} ; B_{p, r}^{s-1, \infty}\right)} \\
& +\frac{1}{4}\left\|\delta \theta^{m}\right\|_{L^{\infty}\left(0, T_{1} ; B_{p, r}^{s-1, \infty}\right)}
\end{aligned}
$$

which yields

$$
\left\|\delta \theta^{m+1}\right\|_{L^{\infty}\left(0, T ; B_{p, r}^{s-1, \infty}\right)} \leq \frac{C_{1} C}{2^{m}}, \text { for } m=0,1,2, \cdots .
$$

Since $\left.\theta^{m}=\theta^{0}+\sum_{k=1}^{m} \delta \theta^{k}, 3.26\right)$ yields a limit $v \in C\left(\left[0, T_{1}\right] ; B_{p, r}^{s-1, \infty}\left(\mathbb{R}^{2}\right)\right.$ such that $\theta^{m}(t) \rightarrow \theta(t)$ uniformly for $t \in\left[0, T_{1}\right]$ in $B_{p, r}^{s-1, \infty}\left(\mathbb{R}^{2}\right)$. Moreover, it is easy to see that $\theta$ is a solution of (1.1) and that, in fact, $\theta \in C\left(\left[0, T_{1}\right] ; B_{p, r}^{s, \infty}\left(\mathbb{R}^{2}\right)\right)$.

Step 4: Uniqueness.

Assume that $\theta_{1}$ and $\theta_{2}$ are two solutions of the system 11.1 with the same initial data $\theta_{0}$ in the class $C\left(\left[0, T_{1}\right] ; B_{p, r}^{s, \infty}\left(\mathbb{R}^{2}\right)\right)$. Denote

$$
\delta \theta=\theta_{2}-\theta_{1}, \quad \delta u=u_{2}-u_{1} .
$$

Then, it follows that $\delta \theta$ satisfies the following system: 


$$
\begin{cases}\partial_{t} \delta \theta+u \cdot \nabla \delta \theta=-\delta u \cdot \nabla \theta, & (x, t) \in \mathbb{R}^{2} \times\left(0, T_{1}\right), \\ u=\mathcal{C}(\theta)+\mathcal{S}(\theta), & (x, t) \in \mathbb{R}^{2} \times\left(0, T_{1}\right), \\ \nabla \cdot \delta u=0, & (x, t) \in \mathbb{R}^{2} \times\left(0, T_{1}\right), \\ \delta \theta(x, 0)=0, & x \in \mathbb{R}^{2} .\end{cases}
$$

We follow the strategy used to derive (3.25) to obtain

$$
\sup _{t \in[0, T]}\|\delta \theta(t)\|_{B_{p, r}^{s-1, \infty}} \leq C T \sup _{t \in[0, T]}\|\delta \theta(t)\|_{B_{p, r}^{s-1, \infty}}
$$

for any $T \leq T_{1}$. Whenever $T$ is small enough such that $C T<1$, we have $\delta \theta(x, t) \equiv 0$ for any $t \leq T$, i.e., $\theta_{2}(x, t) \equiv \theta_{1}(x, t)$. By using the standard continuity argument, we see $\theta_{2}(x, t) \equiv \theta_{1}(x, t)$ for any $t \leq T_{1}$.

Step 5: Blow-up criterion.

From a priori estimate $(3.8)$, we only need to dominate $\|\nabla u\|_{\infty}$ and $\|\nabla \theta\|_{\infty}$. Indeed, it follows from the logarithmic inequality 2.2 with $s>\frac{2}{p}+1$ that

$$
\begin{aligned}
\|\nabla u\|_{\infty} & \leq C\left\{1+\|\nabla u\|_{\dot{B}_{\infty, \infty}^{0}}\left(\log ^{+}\|\nabla u\|_{B_{p, r}^{s-1, \infty}}+1\right)\right\} \\
& \leq C\left\{1+\|\nabla \theta\|_{\dot{B}_{\infty, \infty}^{0}}\left(\log ^{+}\|\theta\|_{B_{p, r}^{s, \infty}}+1\right)\right\},
\end{aligned}
$$

where we used the boundedness of singular integral operator in $\dot{B}_{\infty, \infty}^{0}$. Similarly,

$$
\|\nabla \theta\|_{\infty} \leq C\left\{1+\|\nabla \theta\|_{\dot{B}_{\infty, \infty}^{0}}\left(\log ^{+}\|\theta\|_{B_{p, r}^{s, \infty}}+1\right)\right\} .
$$

Thus, a priori estimate 3.8 gives that

$$
\|\theta\|_{B_{p, r}^{s, \infty}} \leq C\left\|\theta_{0}\right\|_{B_{p, r}^{s, \infty}} \exp \left(C \int_{0}^{t} 1+\|\nabla \theta\|_{\dot{B}_{\infty, \infty}^{0}}\left(\log ^{+}\|\theta\|_{B_{p, r}^{s, \infty}}+1\right) d \tau\right),
$$

which implies that

$$
\|\theta\|_{B_{p, r}^{s, \infty}} \leq C\left\|\theta_{0}\right\|_{B_{p, r}^{s, \infty}} \exp \left(C \exp \left(C \int_{0}^{t}\left(1+\|\nabla \theta\|_{\dot{B}_{\infty, \infty}^{0}}\right) d \tau\right)\right)
$$

by Gronwall's inequality, which implies the desired result for $s>2 / p+1$.

Next, we consider the critical case. Since the embedding $\dot{B}_{\infty, 1}^{0}\left(\mathbb{R}^{2}\right) \hookrightarrow L^{\infty}\left(\mathbb{R}^{2}\right)$ holds, and our singular integral operator is bounded from $\dot{B}_{\infty, 1}^{0}\left(\mathbb{R}^{2}\right)$ into itself, we have

$$
\|\nabla u\|_{\infty} \leq\|\nabla u\|_{\dot{B}_{\infty, 1}^{0}} \leq\|\nabla \theta\|_{\dot{B}_{\infty, 1}^{0}}, \quad\|\nabla \theta\|_{\infty} \leq\|\nabla \theta\|_{\dot{B}_{\infty, 1}^{0}} .
$$

Thus, a priori estimate 3.8 gives that

$$
\|\theta\|_{B_{p, r}^{s, \infty}} \leq C\left\|\theta_{0}\right\|_{B_{p, r}^{s, \infty}} \exp \left(C \int_{0}^{t}\|\nabla \theta\|_{\dot{B}_{\infty, 1}^{0}} d \tau\right),
$$

which implies the result for $s=2 / p+1$. This completes the proof of Theorem 1.1

\section{Acknowledgment}

The authors are grateful to the anonymous referee for his/her careful reading and valuable comments on the manuscript. The work was supported by the National Natural Science Foundation of China (11501332, 11171034), the Natural Science Foundation of Shandong Province (ZR2015AL007), China Postdoctoral Science Foundation funded project (2014M561893), Postdoctoral innovation fund of Shandong Province, the Open Fund of State Key Laboratory of Simulation and Regulation of Water Cycle in River Basin, China Institute of Water Resources and Hydropower Research Fund (IWHR-SKL-201407), the Specialized Research Foundation for the Doctoral Program of Higher Education of China (20123705110001), and the Project of shandong Province Higher Educational Science and Technology Program (J15LI55,J16L101). 


\section{References}

[1] J. Beale, T. Kato, A. Majda, Remark on the breakdown of smooth solutions for the 3-D Euler equations, Comm. Math. Phys., 94 (1984), 61-66. 2

[2] J. Bear, Dynamics of Fluids in Porous Media, Elsevier, New York, (1972). 1 ]

[3] J. Bergh, J. Löfstrom, Interpolation Spaces, An Introduction, Springer-Verlag, Berlin-New York, (1976). 2

[4] D. Chae, On the well-posedness of the Euler equations in the Triebel-Lizorkin spaces, Comm. Pure Appl. Math., 55 (2002), 654-678. 1

[5] D. Chae, On the Euler equations in the critical Triebel-Lizorkin spaces, Arch. Rational Mech. Anal., 170 (2003), 185-210. 1

[6] D. Chae, Local existence and blow-up criterion for the Euler equations in the Besov spaces, Asymptot. Anal., 38 (2004), 339-358.1

[7] J.-Y. Chemin, Perfect incompressible fluids, Oxford Lectures Series in Mathematics and its Applications, The Clarendon Press, Oxford University Press, New York, (1998). 2.4, 2.5

[8] D. Córdoba, F. Gancedo, R. Orive, Analytical behavior of the two-dimensional incompressible flow in porous media, J. Math. Phys., 48 (2007), 19 pages. 1, 1.2

[9] D. Nield, A. Bejan, Convection in Porous Media, Springer-Verlag, New York, (1999).1

[10] M. Price, Introducing Groundwater, 2nd ed. Chapman and Hall, London, (1996). 1 ]

[11] E. M. Stein, Harmonic Analysis, Princeton University Press, Princeton, NJ, (1993). 1

[12] R. Takada, Local existence and blow-up criterion for the Euler equations in Besov spaces of weak type, J. Evol. Equ., 8 (2008), 693-725.1, 2.8, 2.9,2.10, 2.12, 2.13

[13] W. Yu, Y. He, On the well-posedness of the incompressible porous media equation in Triebel-Lizorkin spaces, Boundary Value Problems, 2014 (2014), 11 pages. 1 